\title{
Bariatric Surgery and Diabetes: Access Denied
}

\author{
William H. Chapman, Emily Cunningham, and Walter J. Pories
}

\section{Introduction}

$\mathrm{T}$ HIS YEAR, FOR THE FIRST TIME, we have been provided with strong proof that bariatric surgery is truly effective. Over the last 30 years, there have been multiple reports that the gastric bypass operation provides full remission of diabetes in four out of five people, with a sharp decrease in mortality. Along with the reversal of diabetes, most of those who receive the surgery will also see their high blood pressure, sleep apnea, dyslipidemias, and even their polycystic ovary syndromes clear. Further, within five years, their likelihood of getting various cancers diminishes by more than $70 \%$. Even those who do not experience full remission of their diabetes show marked improvement in control of their disease. Finally, we have developed and demonstrated the success of various bariatric procedures (lap adjustable BAND, gastric bypass, sleeve gastrectomy, and duodenal switch) that have impressive metabolic consequences beyond the remission of diabetes.

In spite of these excellent outcomes widely reported in the surgical literature, less than one percent of those who could benefit from bariatric surgery have accessed these procedures.

The skepticism of the medical community is not unwarranted. The claims seem unbelievable; the reports are primarily from surgeons, and the past history of bariatric surgery, a long period of trial and error, includes a number of false and dangerous starts. Accordingly, it is appropriate for physicians, concerned about their patients' safety, to ask if bariatric surgery is really effective, and if it is safe and costeffective. This year, supported by several key articles cited below, we can answer these questions affirmatively.

\section{Bariatric surgery versus intensive medical therapy in obese patients with diabetes}

Schauer $P R^{1}$, Kashyap $S R^{2}$, Wolski $K^{3}$, Brethauer $S A^{1}$, Kirwan $J P^{4}$, Pothier $C E^{3}$, Thomas $S^{1}$, Abood $B^{1}$, Nissen $S E^{5}$, Bhatt $D L^{6}$

${ }^{1}$ Bariatric and Metabolic Institute, Cleveland Clinic, Cleveland, $\mathrm{OH} ;{ }^{2}$ Endocrinology Institute, Cleveland Clinic, Cleveland, $\mathrm{OH}$; ${ }^{3}$ Cleveland Clinic Coordinating Center for Clinical Research, Cleveland Clinic, Cleveland, $\mathrm{OH} ;{ }^{4}$ Heart and Vascular Institute, Cleveland Clinic, Cleveland, $\mathrm{OH} ;{ }^{5}$ Lerner Research Institute,
Cleveland Clinic, Cleveland, $\mathrm{OH} ;{ }^{6}$ Veterans Affairs Boston Healthcare System, Bringham and Women's Hospital and Harvard Medical School, Boston, MA

N Engl J Med 2012; 366: 1567-76

\section{Background}

Although multiple series have been published documenting the effectiveness of bariatric surgery in the treatment of T2DM, there were no credible prospective randomized series.

\section{Methods}

150 obese patients, $66 \%$ women, with uncontrolled T2DM with a mean $\mathrm{Hb} 1 \mathrm{Ac}$ of $9.2 \pm 1.55 \%$ were prospectively randomized to intensive medical therapy, Roux-en- $Y$ gastric bypass, or sleeve gastrectomy.

\section{Results}

The proportion of patients in this 12-month study who completed the follow-up was $93 \%$. The proportion of patients with the primary endpoint $(\mathrm{Hb} 1 \mathrm{Ac}=6.0 \%)$ was $12 \%$ (5 of 41 patients) in the medical-therapy group vs. $42 \%$ (21 of 50 patients) in the gastric-bypass group $(p=0.002)$ and $37 \%$ (18 of 49 patients) in the sleeve-gastrectomy group ( $p=0.008)$. Glycemic control improved in all three groups, with a mean glycated hemoglobin level of $7.5 \pm 1.8 \%$ in the medicaltherapy group, $6.4 \pm 0.9 \%$ in the gastric-bypass group $(\mathrm{p}<0.001)$, and $6.6 \pm 1.0 \%$ in the sleeve-gastrectomy group $(p=0.003)$.

\section{Conclusions}

Bariatric surgery is more effective than intensive medical therapy for the treatment of obese patients with uncontrolled T2DM.

\section{Bariatric surgery versus conventional medical therapy for type 2 diabetes}

Mingrone $G^{1}$, Panunzi $S^{2}$, De Gaetano $A^{2}$, Guidone $C^{1}$, Iaconelli $A^{1}$, Leccesi $L$, Nanni $G^{3}$, Pomp $A^{4}$, Castagneto $M^{3}$, Ghirlanda G, Rubino $F^{3,4}$ 
${ }^{1}$ Department of Internal Medicine, Università Catolica S. Rome, Italy; ${ }^{2}$ Institute of Systems Analysis and Computer Science (IASI) and BioMatLab, Rome, Italy; ${ }^{3}$ Department of Surgery, Università Catolica S. Rome, Italy; and ${ }^{4}$ Section of Gastrointestinal Metabolic Surgery, Weill Medical College of Cornell University, New York Presbyterian Hospital, New York, NY

N Engl J Med 2012; 366: 1577-85

\section{Background}

Prospective randomized trials are needed to answer the question if bariatric surgery is more effective in the treatment of T2DM than intensive medical therapy.

\section{Methods}

Sixty patients ages $30-60, \mathrm{BMI} \geq 35$, with a history of at least five years of T2DM, were prospectively randomized to participate in conventional medical therapy or undergo either gastric bypass or biliopancreatic diversion.

\section{Results}

At two years, diabetes remission had occurred in no patients in the medical-therapy group vs. $75 \%$ in the gastricbypass group and $95 \%$ in the biliopancreatic-diversion group ( $\mathrm{p}<0.001$ for both comparisons). Also at two years, the average baseline glycated hemoglobin level $(8.65 \pm 1.45 \%)$ had decreased in all groups, but patients in the two surgical groups had the greatest degree of improvement (average glycated hemoglobin levels, $7.69 \pm 0.57 \%$ in the medicaltherapy group, $6.35 \pm 1.42 \%$ in the gastric-bypass group, and $4.95 \pm 0.49 \%$ in the biliopancreatic-diversion group).

\section{Conclusions}

Bariatric surgery was significantly more effective in the control of weight and T2DM than medical therapy.

\section{Bariatric surgery is associated with a reduced risk of mortality in morbidly obese patients with a history of major cardiovascular events}

Johnson RJ, Johnson BL, Blackhurst DW, Bour ES, Cobb WS 4th, Carbonell AM 2nd, Lokey JS, Scott JD

Greenville Hospital System University Medical Center, Greenville, South Carolina

Am Surg 2012; 78: 685-92

\section{Background}

There is only spare data about the long-term survival in patients with cardiovascular disease after bariatric surgery.

\section{Methods}

These authors reported a retrospective cohort study of 349 bariatric surgical patients vs. 903 morbidly obese controls with a history of cardiovascular events.

\section{Results}

Among bariatric patients, 19 deaths occurred in 986 personyears of follow-up vs. 150 deaths among controls in 3,138 person-years of follow-up. Adjusting for age, comorbidity, and event history, the relative risk of mortality was reduced by $40 \%$ in bariatric patients compared with controls (hazard ratios [95\% confidence interval]: 0.60 [0.36, 0.99]). Unadjusted all-cause mortality was estimated at $7 \pm 2 \%$ at 5 years in bariatric patients compared with $19 \pm 2 \%$ in controls $(p<0.001)$.

\section{Conclusion}

In patients with a history of cardiovascular events, bariatric surgery is associated with a significantly decreased risk of allcause mortality.

\section{Comment}

Is the correction of glucose levels by bariatric surgery really important? Does it make a difference in mortality and morbidity?

It may seem curious to ask whether the correction of glucose levels in T2DM is a useful goal, but there is merit in the concern that the correction of glucose laboratory levels cannot be extrapolated to long-term health effects. Actually, this question was addressed before by MacDonald et al. (1), who reported that diabetic patients who underwent the gastric bypass had mortality rates $73 \%$ lower than a comparable group who did not. The study by Johnson et al. confirms that finding.

The answer is clear: Bariatric surgery does reduce mortality in a very high-risk patient cohort. The improvement/remission of diabetes, decrease in hypertension, improved lipid profile, and other still unknown metabolic changes that occur with bariatric surgery all contribute to improved longevity.

\section{Perioperative safety and volume: outcomes relationships in bariatric surgery: a study of 32,000 patients}

Gould $\mathrm{JC}^{1}$, Kent $K \mathrm{C}^{2}$, Wan $Y^{2}$, Rajamanickam $V^{2}$, Leverson $G^{2}$, Campos $\mathrm{GM}^{2}$

${ }^{1}$ Medical College of Wisconsin, Department of Surgery, Milwaukee, WI; and ${ }^{2}$ University of Wisconsin School of Medicine and Public Health, Department of Surgery, Madison, WI

J Am Coll Surg 2011; 213: 771-7

\section{Background}

There is no evidence-based data about the association between the amount of yearly bariatric surgical procedures and the medical outcomes.

\section{Methods}

The nationwide inpatient sample, 2005-2007, was queried for open and laparoscopic bariatric procedures, complications, and death.

\section{Results}

A total of 32,509 bariatric procedures were identified (21\% open bypass [Open], 58\% laparoscopic bypass [Lap], 21\% laparoscopic gastric band [Band]). Inpatient overall mortality was low (total 0.12\%, Open 0.3\%, Lap 0.09\%, Band 0.02\%; p $<0.05$ for all comparisons). Inpatient complications were more prevalent in open procedures (total 3.9\%, Open 5.9\%, Lap 4\%, Band 1.6\%; 
$\mathrm{p}<0.01$ for all comparisons). For all three procedures, using a combined endpoint of mortality and major complications, a volume-outcomes relationship was evident for hospitals.

\section{Conclusions}

The nationwide dataset and bariatric procedure-specific data documents that bariatric surgery mortality and complication rates are very low.

\section{Comment}

How safe is bariatric surgery?

With the basic rule of medicine, i.e., "primum nocere," it is mandatory that each of our therapies be carefully examined in terms of safety. This is especially true in the assessment of surgery for the severely obese, a group of patients that all too often enter the operating rooms with anesthesia risk evaluations of three and four due to their comorbidities.

This article reflects the remarkable advances in the safe delivery of bariatric surgery. In the beginning of this century, mortality rates of $10 \%$ were not uncommon, and the press was filled with horror stories about "stapled stomachs" and lawsuits. With the advent of laparoscopic procedures, improved equipment and technology, and improved perioperative evaluation and care, the morbidity and mortality of bariatric procedures have diminished. The development of Centers of Excellence by the Association of Metabolic and Bariatric Surgeons (ASMBS), the Surgical Review Corporation (SRC), the American College of Surgeons (ACS), and the National Surgical Quality Improvement Program (NSQIP) has been crucial to insuring good quality programs and outcomes to the point where bariatric surgery is now delivered in over 450 U.S. hospitals with a 90 -day mortality of $0.3 \%$, equal to that reported for routine cholecystectomies even though the morbidly severe present with far greater risks.

\section{Healthcare utilization and costs in severely obese subjects before bariatric surgery}

Keating $C L^{1}$, Moodie $M L^{1}$, Bulfone $L^{1}$, Swinburn $B A^{2}$, Stevenson $C E^{3}$, Peeters $A^{3,4}$

${ }^{1}$ Deakin Health Economics, Deakin University, Melbourne, Australia; ${ }^{2}$ World Health Organization Collaborating Centre for Obesity Prevention, Deakin University, Melbourne, Australia; ${ }^{3}$ Department of Epidemiology and Preventive Medicine, Monash University, Melbourne, Australia; and ${ }^{4}$ Obesity and Population Health, Baker IDI, Heart and Diabetes Institute, Melbourne, Australia

Obesity (Silver Spring) 2012 May 4: [Epub ahead of print]; DOI: 10.1038/oby.2012.124

\section{Background}

It is important to evaluate whether bariatric surgery is costeffective.

\section{Methods}

This study evaluated the healthcare utilization and associated costs for a severely obese population before receiving laparoscopic adjustable gastric banding (LAGB) surgery in $2009(n=11,769)$ relative to an age- and sex-matched sample from the Australian general population. Utilization of medical services and pharmaceuticals in the 3.5 years before surgery were ascertained for each severely obese subject through linkage with Medicare, Australia's universal health insurance scheme. Equivalent data were retrieved for each subject from the matched general population sample $(n=140,000)$.

\section{Results}

Severely obese subjects utilized significantly more medical services annually compared to the general population (mean: 22.8 vs. 12.1 /person, standardized incidence ratio [SIR]: 1.89 [95\% confidence interval (CI) 1.88-1.89]), translating to two-fold higher mean annual costs (Australian $\$ 1,140$ vs. \$567/person). The greatest excess costs in the obese related to diabetes drugs, lipid-modifying agents, psychoanaleptics, acid-related disorder drugs, agents acting on the rennin-angiotensin system, immune suppressants, and obstructive airway disease drugs.

\section{Conclusion}

Overall, healthcare costs in the severely obese population were more than double those incurred by the general population.

\section{Early postoperative outcomes and medication cost savings after laparoscopic sleeve gastrectomy in morbidly obese patients with type 2 diabetes}

Slater $B J^{1}$, Bellatorre $N^{2}$, Eisenberg $D^{1,2}$

${ }^{1}$ Department of Surgery, Stanford University School of Medicine, Stanford, CA; and ${ }^{2}$ Department of Surgery, VA Palo Alto Health Care System, Palo Alto, CA

J Obes 2011 Dec 7; 2011: [Epub ahead of print]; DOI:10.1155/2011/ 350523

\section{Background}

Evaluation of the short-time effect of laparoscopic sleeve gastrectomy (LSG) in obese patients with T2D is mandatory.

\section{Methods}

In a prospective study of 178 patients subjected to LSG, age, gender, body mass index (BMI), diabetic medication use, glucose, insulin, and $\mathrm{HbA1c}$ levels were documented preoperatively and at 2 weeks, 2 months, 6 months, and 12 months postoperatively. Use and cost of diabetic medications were followed.

\section{Results}

Of 178 patients, 22 were diabetics who underwent LSG. Diabetes remission was observed in $62 \%$ of patients within 2 months and in $75 \%$ of patients within 12 months. Average number of diabetic medications decreased from 2.2 to $<1$ within 2 weeks after surgery; corresponding to a diabetes medication cost savings of $80 \%, 91 \%, 99 \%$, and $99.7 \%$ after 2 weeks, 2 months, 6 months, and 12 months, respectively.

\section{Conclusion}

Patients with T2D and morbid obesity who undergo LSG have high rates of diabetes remission early after surgery, with a significant medication cost savings. 
Incorporating indirect costs into a cost-benefit analysis of laparoscopic adjustable gastric banding

Finkelstein $E A^{1}$, Allaire $B T^{2}$, Dibonaventura $\mathrm{MD}^{3}$, Burgess $S M^{4}$

${ }^{1}$ Duke-NUS Graduate Medical School, Singapore; ${ }^{2}$ RTI International, Research Triangle Park, NC; ${ }^{3}$ Health Sciences Practice, Kantar Health, New York, NY; and ${ }^{4}$ Global Health Outcomes Strategy and Research, Allergan Inc., Irvine, CA

Value Health 2012; 15: 299-304

\section{Background}

This study estimated the time to breakeven and the fiveyear net costs of laparoscopic adjustable gastric banding (LAGB), taking into account both direct and indirect costs and cost savings from the literature.

\section{Methods}

Total savings were calculated based on quantifying relationships between medical expenditures, absenteeism, and presenteeism. These savings were then combined with the direct and indirect costs to quantify net savings.

\section{Results}

Including indirect costs after five years, net savings in medical expenditures from a gastric banding procedure were estimated to be $\$ 4,970( \pm \$ 3,090)$. Including absenteeism increased savings to $\$ 6,180( \pm \$ 3,550)$. Savings were further increased to $\$ 10,960( \pm \$ 5,864)$ when both absenteeism and presenteeism estimates were included.

\section{Conclusions}

This is an important contribution because the authors examined more than direct costs but included improvements in performance at work, noting that inclusion of these data documented even greater cost advantages for bariatric surgery.

\section{Impact of bariatric surgery on health care utilization and costs among patients with diabetes}

Bleich $S N^{1}$, Chang $H Y^{1}$, Lau $B^{2,3}$, Steele $K^{4}$, Clark $J M^{2,3}$,

Richards $T^{1}$, Weiner $J P^{1}, W u A W^{1,2,3,4}$, Segal $J B^{1,2,3}$

${ }^{1}$ Department of Health Policy and Management, ${ }^{2}$ Department of Epidemiology, ${ }^{3}$ Department of Medicine, ${ }^{4}$ Department of Surgery, The Johns Hopkins University School of Medicine, Baltimore, $M D$

Med Care 2012; 50: 58-65

\section{Background}

There is no clear data about the impact of bariatric surgery on healthcare utilization in patients with T2D.

\section{Methods}

Healthcare utilization and costs in an insured cohort of 7,806 individuals with T2D after bariatric surgery were derived from administrative data from 2002 to 2008 from seven Blue Cross Blue Shield Plans.

\section{Results}

If the cost of the bariatric surgery was excluded, hospital costs were higher in year two after surgery. However, in terms of having any healthcare costs, those costs were lower after surgery as well. However for those requiring hospitalization, the ratio of inpatient days was higher.

\section{Conclusion}

Individuals with T2D did not have lower healthcare costs in the six years after surgery.

\section{Comment}

\section{Is bariatric surgery cost-effective?}

The third essential element for a successful medical therapy, in addition to efficacy and safety, is "efficiency" (i.e., the use of resources and/or "cost"). However, evaluation of cost is difficult due to the many confounding variables: 1) charges do not necessarily reflect costs; 2 ) costs and outcomes vary by region, by hospitals, by providers, by carriers, by age, by race, by the economic and educational status of the patients, by the stages of the diseases and co-morbidities; and 3) it is difficult to determine the costs and values of favorable results such as (a) the reduction of disabilities due to the loss of weight, (b) the value of return to employment or studies, and (c) the pursuit of remedial procedures, such as hip replacement, transplant, or plastic surgery. For example, how can one calculate the cost and value of the gastric bypass performed on Mr. Al Roker, the popular WNBCTV weatherman who, reportedly, was only able to climb three steps due to his weight and heart failure prior to his operation on March 22, 2002? He is now able to pursue a highly successful and well-paid career.

The above four important articles have added to our knowledge about the economics of obesity and bariatric surgery this year. The first three papers support the general impression reflected in the literature over the last 10 years, that bariatric surgery reduces healthcare expenditures with a return of the costs over the following two to three years. The fourth paper documents again how difficult these studies are. The BCBS study was limited to in-hospital costs and failed to address the considerable costs incurred from knee replacements, hip replacements, plastic surgery, and other corrective operations that only became possible after bariatric surgery.

\section{Physicians' attitudes about referring their type 2 diabetes patients for bariatric surgery}

Sarwer $D B^{1,2}$, Ritter $S^{1}$, Wadden $T A^{1}$, Spitzer $J C^{1}$, Vetter $M L^{3}$, Moore $\mathrm{RH}^{1,4}$

${ }^{1}$ Department of Psychiatry, ${ }^{2}$ Department of Surgery, ${ }^{3}$ Department of Medicine, ${ }^{4}$ Department of Biostatistics and Epidemiology, University of Pennsylvania Perelman School of Medicine, Philadelphia, PA

Surg Obes Relat Dis 2012; Jul-Aug; 8: 381-6

\section{Background}

Little data is available about the physician's attitudes toward treatment of bariatric surgery in patients with T2D. 


\section{Methods}

Physicians at an academic medical center $(n=142)$ and community-based physicians $(n=197)$ in the Philadelphia area in specialties likely to treat T2D were sent a survey about their perceptions of the safety and efficacy of bariatric surgery as a treatment for obesity and T2D.

\section{Results}

With a combined response rate of $27.4 \%$, physicians reported having positive impressions of bariatric surgery as a treatment for obesity and T2D $(79.6 \%$ and $67.4 \%$, respectively). Yet only $20.8 \%$ of respondents indicated that they would be likely to refer their patients with T2D with a BMI of 30$34.9 \mathrm{~kg} / \mathrm{m}^{2}$ to a randomized research trial of bariatric surgery.

\section{Conclusions}

Physicians who treat patients with T2D and obesity had favorable impressions about bariatric surgery as a treatment. However, only a small percentage of them were willing to refer the patients with $\mathrm{T} 2 \mathrm{D}$ and obesity to randomized research trials of bariatric surgery. This reflects an important barrier to the successful completion of studies of the efficacy of bariatric surgery for those with T2D and a BMI $<35 \mathrm{~kg} / \mathrm{m}^{2}$.

\section{Comment}

Why do less than one percent of those who would benefit utilize bariatric surgery?

The low utilization of bariatric surgery (i.e., less than 1\% of the patients who could benefit undergo the surgery) continues to be a puzzle. The reason for lack of utilization of bariatric surgery is multifactorial. In our experience, physicians' attitudes are only a part of the challenge. Patients, themselves, have an aversion to undergoing surgery, especially to avert the complications of T2DM that may not appear for another decade. Also, many patients are frightened of the disasters of bariatric surgery that are reported in numerous media outlets, not understanding that the majority of outcomes are far different. The biggest hurdles, however, are the difficulties and delays in obtaining coverage by insurance carriers, often with requirements that are completely unfounded by the current literature, such as demands that the morbidly obese patients lose significant weight prior to getting approval for bariatric surgery. In patients who have unsuccessfully tried dieting for years, it is not surprising that many fail this cruel test and just give up.
Medicaid coverage has also been withdrawn in a number of states due to budget problems and perhaps bias against obesity, even though these operations are cost-effective.

\section{Conclusions}

It has taken over a half century to develop bariatric surgery to the point where even the original name, based on "baros" for weight, no longer fits. Although the four currently accepted procedures (the adjustable gastric band, the gastric sleeve, the gastric bypass, and the biliopancreatic bypass with duodenal switch) are indeed the most effective therapies for weight control, they also produce far more important health effects that include the remission of type 2 diabetes, hypertension, nonalcoholic steatohepatitis (NASH), gastroesophageal reflux (GERD), polycystic ovary syndrome (PCOS), asthma, cardiopulmonary failure, and even the sharp reduction in the prevalence of cancer. Accordingly, the new proposed name, "metabolic surgery," offers a far more fitting description.

The selected papers this year have made an important contribution. Together with other publications not noted here, the literature now offers strong support for the conclusion that bariatric surgery is effective; in fact, more effective than current medical therapies. In addition, the data show that the surgery is safe and cost-effective.

While this approbation of bariatric surgery is important, it is really only the start of the journey. We cannot announce success until we understand the pathways of the metabolic syndrome, until we can abandon surgical approaches for medical measures, and, even better, prevent the diseases. We have not completed our task. Although we have made significant strides to understanding the physiologic and metabolic changes that occur after bariatric surgery, we have a long way to go!

\section{Author Disclosure Statement}

W.H.C. and E.C. declare no potential conflicts of interest. W.J.P. receives grant support from the NIDDK/National Institutes of Health, Johnson \& Johnson, and Glaxo SK.

\section{Reference}

1. MacDonald, KG, Long, SD, Swanson, MS, Brown, BM, Morris, PG, Dohm, GL and Pories, WJ. "The Gastric Bypass Operation Reduces the Progression and Mortality of Non-Insulin Dependent Diabetes Mellitus." Journal of Gastrointestinal Surgery. 1: 213-220, 1997. 